Article

\title{
Attributable Mortality of Hip Fracture in Older Patients: A Retrospective Observational Study
}

\author{
Lorène Zerah 1,2,*, David Hajage ${ }^{2,3}$, Mathieu Raux ${ }^{4,5}$, Judith Cohen-Bittan ${ }^{1}$, Anthony Mézière ${ }^{6}$, \\ Frédéric Khiami ${ }^{7}$, Yannick Le Manach ${ }^{8}$, Bruno Riou ${ }^{9,10}$ and Jacques Boddaert ${ }^{1,11}$ \\ 1 Department of Geriatric Medicine, Hôpital la Pitié-Salpêtrière, Assistance Publique-Hôpitaux de \\ Paris (APHP), 75013 Paris, France; judith.cohen-bittan@aphp.fr (J.C.-B.); jacques.boddaert@aphp.fr (J.B.) \\ 2 UMRS INSERM 1136, Institut Pierre Louis d'Epidémiologie et de Santé Publique, Sorbonne Université, \\ 75013 Paris, France; david.hajage@aphp.fr \\ 3 Department of Biostatistics, Hôpital la Pitié-Salpêtrière, APHP, 75013 Paris, France \\ 4 UMRS INSERM 1158, Sorbonne Université, 75013 Paris, France; mathieu.raux@aphp.fr \\ Department of Anesthesiology and Critical Care, Hôpital la Pitié-Salpêtrière, APHP, 75013 Paris, France \\ 6 Department of Rehabilitation, Hôpital Charles Foix, APHP, 94200 Ivry sur Seine, France; \\ anthony.meziere@aphp.fr \\ 7 Department of Orthopedic Surgery and Trauma, Hôpital La Pitié-Salpêtrière, APHP, 75013 Paris, France; \\ Frederic.khiami@aphp.fr \\ 8 Departments of Anesthesia \& Health Research Methods, Evidence \& Impact (HEI), Michael DeGroote School \\ of Medicine, Faculty of Health Sciences, McMaster University, Hamilton, ON L8S 4L8, Canada; \\ yannick.lemanach@phri.ca \\ 9 UMRS INSERM 1166, IHU ICAN, Sorbonne Université, 75013 Paris, France; bruno.riou@aphp.fr \\ 10 Department of Emergency Medicine and Surgery, Hôpital La Pitié-Salpêtrière, APHP, 75013 Paris, France \\ 11 UMR INSERM U1135, Sorbonne Université, 75013 Paris, France \\ * Correspondence: lorene.zerah@inserm.fr
}

Received: 16 June 2020; Accepted: 23 July 2020; Published: 24 July 2020

check for updates

\begin{abstract}
Hip fracture (HF) in older patients is associated with a high six-month mortality rate. Several clinical conditions may affect outcome, including baseline characteristics, co-existing acute illnesses, perioperative factors, and postoperative complications. Our primary objective was to estimate the respective effect of these four domains on six-month mortality after HF. A retrospective observational study using a monocentric cohort of older patients was conducted. All patients $\geq$ 70 years old admitted to the emergency department for HF and hospitalized in our perioperative geriatric care unit from June 2009 to September 2018 were included. Among 1015 included patients, five $(0.5 \%)$ were lost to follow-up, and 1010 were retained in the final analysis (mean age $86 \pm 6$ years). The six-month mortality rate was $14.8 \%$. The six-month attributable mortality estimates were as follows: baseline characteristics (including age, gender, comorbidities, autonomy, type of fracture): $62.4 \%$; co-existing acute illnesses (including acute events present before surgery that could result from the fracture or cause it): $0 \%$ (not significantly associated with six-month mortality); perioperative factors (including blood transfusion and delayed surgery): $12.3 \%$; severe postoperative complications: $11.9 \%$. Baseline characteristics explained less than two-thirds of the six-month mortality after HF. Optimizing patients care by improving management of perioperative factors and thus decreasing postoperative complications, could reduce by a maximum of one quarter of the six-month mortality rate after HF.
\end{abstract}

Keywords: hip fracture; attributable risk; mortality; prognosis; elderly 


\section{Introduction}

Hip fracture (HF) is a major public health concern that has increasing socio-economic consequences [1]. Although its incidence is decreasing, the total number of HF cases is increasing in line with the aging population (more than 1.6 million people undergo hip fracture in the world every year) [2,3]. This frequent emergency condition is associated with a poor prognosis. Mortality is much higher in people after HF than in the general population with comparable age, and remains high for many months after HF [4]. Mortality rate at six months varies from 8 to $26 \%$ [5].

As compared with elective total hip replacements, for HF patients, the mortality is increased 6- to 15-fold [6]. This difference was thought to be explained by the high prevalence of pre-existing medical conditions in this population, which is known to be associated with poor medical outcomes [2,7-9]. However, even when patients are matched for age, sex, and preoperative comorbidities, the in-hospital mortality after HF remains six times higher than that observed after elective total hip replacement [10]. Moreover, we previously found that geriatric scores, including age, sex, and comorbidities gave poor results to predict short- (30 days) and long-term (six months) postoperative mortality [11]. We can assume that factors other than baseline characteristics have a great impact on survival, including, for example, delayed surgery, anesthesia type, transfusions, or postoperative delirium [7-9,12]. The demonstration that a dedicated clinical action plan can significantly improve the six-month mortality of elderly patients with HF, compared to patients admitted to the orthopedic surgery department, is in favor of this hypothesis [9]. However, we searched on PubMed, on 1st March, 2018 and on 1st December, 2019, if a study had attempted to quantify the attributable mortality of HF in the elderly, without success (((attributable mortality[Title/Abstract]) OR (averaged attributable fractions[Title/Abstract])) AND (hip fracture[Title/Abstract])).

We hypothesized that understanding the respective influence on factors associated to six-month mortality after $\mathrm{HF}$ and identifying the modifiable factors with the highest impact would be an essential step that could indicate the directions for care improvement. We proposed to classify all these factors into four domains: baseline characteristics, co-existing acute illnesses, perioperative factors, and postoperative complications. The relation between these factors and postoperative mortality is complex, and a fuller understanding of the contribution of each factor is needed to develop a better predictive model for HF outcomes in older people.

Our primary objective was to evaluate the respective influence of these four domains on six-month mortality in patients after HF.

\section{Materials and Methods}

The database was declared to the French National Commission on Computing and Liberty (CNIL) of Assistance Publique-Hôpitaux de Paris (APHP) (no 20190426181554). This report follows the STROBE and TRIPOD recommendations (Text S1) [13,14].

\subsection{Study Design, Setting, and Participants}

This retrospective observational study was conducted in the perioperative geriatric unit (UPOG) of an academic hospital. UPOG is part of a dedicated orthogeriatric care pathway defined as follows: (1) early alert from the emergency department (ED); (2) consideration of HF as requiring surgery as soon as feasible (i.e., $24 \mathrm{~h}$ a day); (3) rapid transfer to the UPOG after surgery; and (4) rapid transfer of stable patients to a dedicated rehabilitation unit [8]. The management strategy in UPOG was previously described [9] and is detailed in Text S2.

From June 2009 to September 2018 (111 months), all consecutive patients with HF admitted to the UPOG were evaluated for eligibility. Patients were included if their primary presentation was due to HF (first hospitalization in UPOG) and if they were $\geq 70$ years old. Patients with metastatic or periprosthetic fractures were excluded. Patients were followed until death or six months after admission. Surviving patients or their family were seen in routine consultation at six months or were 
contacted and interviewed by telephone. Missing patients were tracked through health care providers, particularly general practitioners, or any identified acquaintances.

\subsection{Description of the 4 Domains}

We classified factors that could be associated with HF mortality into 4 domains: (1) baseline characteristics (including comorbidities but also age, gender, frailty, chronic medications, autonomy, functional status, and type of fracture); (2) co-existing acute illnesses (including acute events present before surgery that could result from the fracture or cause it); (3) perioperative factors (including delay to surgery, type of anesthesia, hemodynamic stability, and bleeding); and (4) postoperative complications (Figure 1).

\section{MODEL FOR ATTRIBUABLE MORTALITY}

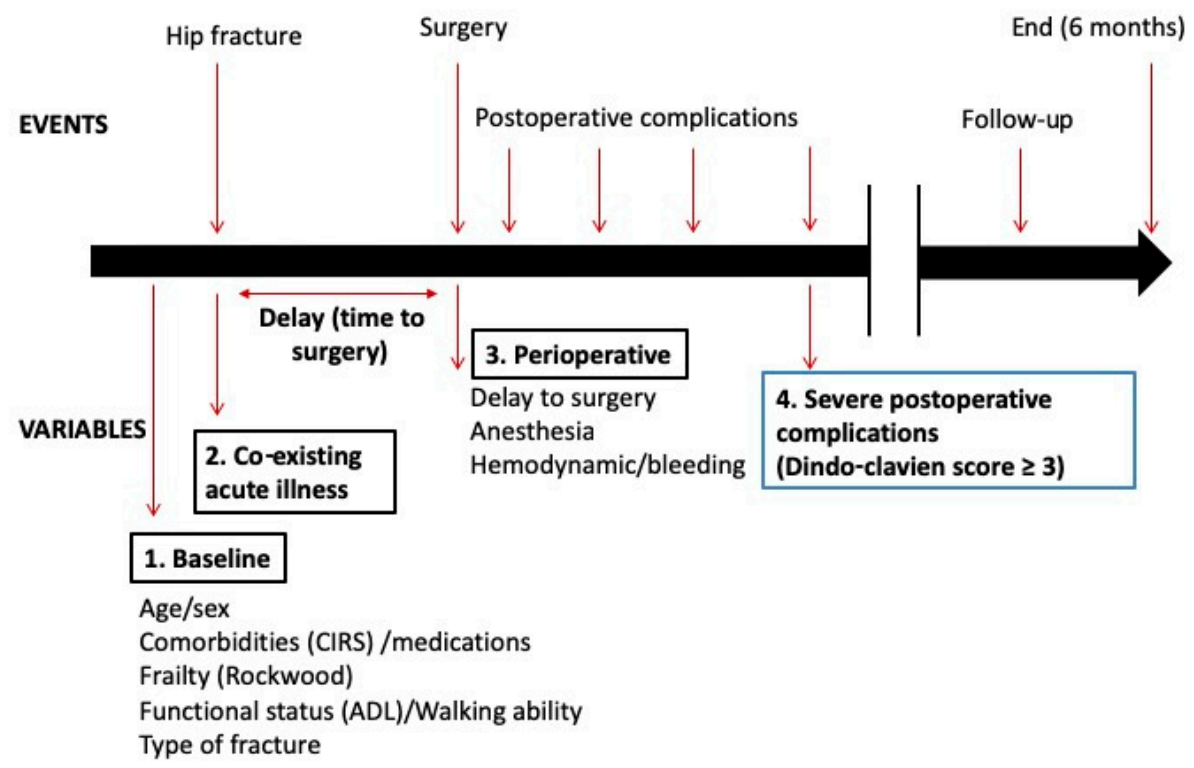

Figure 1. Clinical model for estimating attributable mortality in older patients with hip fracture. Four domains of risk factors (numbered 1 to 4 ) were investigated. Abbreviations: ADL = Activities of Daily Living; CIRS: Cumulative Illness Rating Scale.

\subsection{Data Collection}

Since the opening of UPOG in 2009, we have created a dedicated research database that was prospectively supplemented by 3 senior geriatricians (J.B., J.C.-B., L.Z.), experts in orthogeriatrics, and that integrates all the data from the orthogeriatric care pathway for each patient.

The following variables, collected prospectively, by interviewing patients, their family members or their physicians and pharmacists during the hospital stay, defined baseline characteristics before HF: age, gender, home or nursing home living conditions, walking ability, previous medical history, chronic medications, and type of fracture (radiological definition by an orthopedic surgeon). Co-morbidity severity, frailty, and functional status were calculated prospectively by one of the 3 senior geriatricians during the hospital stay. Co-morbidity severity was assessed with the Cumulative Illness Rating Scale (CIRS) [15], because all comorbidity scores are equivalent in predicting mortality in this population [11]. Frailty was assessed with the Rockwood score [16]. Functional status was evaluated with the Activities of Daily Living (ADL) scale [17] and the Instrumental Activities of Daily Living (IADL) scales [18].

During the perioperative period, we prospectively recorded the surgical treatment, delay and duration of surgery, type of anesthesia provided (general vs. spinal), and the amount of blood transfusion administered. After surgery, delays to first sitting and first walking, the destination 
at discharge of UPOG (home or rehabilitation), and length of stay in acute care and rehabilitation departments were recorded.

All postoperative complications during the acute care period (not in rehabilitation) were prospectively recorded.

Co-existing acute illnesses and the severity of postoperative complications were the only 2 variables to have been classified retrospectively, before any statistical analysis. They were both adjudicated by 2 senior geriatricians (J.B. and L.Z.), independently reviewing the medical charts (Kappa score 0.90 for acute co-existing illnesses and 0.97 for Dindo-Clavien score). In case of disagreement, reconciliation was reached with a third independent senior expert (B.R.).

Co-existing acute illnesses were defined as acute events that could have promoted the fall (e.g., acute coronary syndrome, stroke) or acute events that could result from the fall (e.g., other traumatic lesions, rhabdomyolysis) requiring specific treatment and/or affecting the prognosis. By definition, these conditions had to be present before surgery, but no predefined list was established.

The severity of postoperative complications was adjudicated with the Dindo-Clavien classification [19]. Dindo-Clavien classes from 3 to 5 were considered as severe complications. With several postoperative complications, the highest Dindo-Clavien score defined the final postoperative score.

\subsection{Statistical Analyses}

The statistician who performed the analyses was an independent statistician who was not involved in data collection or the initial definition of study objectives. The statistical plan of the study was established by him, after discussion with the authors, before transmission of the data and the beginning of the analyses (Text S3). As the database was prospectively supplemented, all the authors were "blinded" to the research question at the time of data collection. The study is based on all available patients during the study period and thus no a priori power calculation was conducted.

Data are presented as mean $\pm \mathrm{SD}$, median (25-75 interquartile range) for non-Gaussian distributed variables, or numbers (percentages). Comparison of quantitative variables between survivors and deceased patients involved unpaired Student $t$ tests, or Mann-Whitney tests in case of rejection of the normality assumption in one or both groups. Normality was assessed using Anderson-Darling test. Comparison of categorical variables involved chi-square test or Fisher's exact test, as appropriate. Survival was estimated by the Kaplan-Meier method.

Each explanatory variable was classified a priori according to 4 domains: baseline characteristics, occurrence of co-existing acute illness, perioperative period characteristics, and the occurrence of postoperative complications. To ensure the validity of this classification, the process was conducted independently by 3 physicians with expertise in perioperative care (J.B., L.Z., B.R.). Full consensus between the experts was required to include a variable within a domain. Correlation between continuous variables was considered significant when the Spearman correlation coefficient was $>0.50$. The choice between 2 correlated variables was based on their respective clinical relevance. Then, continuous variables were dichotomized by clinically relevant thresholds from the literature or by receiver operating characteristic curve analysis to determine the best threshold for 6-month mortality after HF (maximization of the Youden index).

Assuming a causal link between exposures and mortality, the sum of the exposures averaged attributable fractions (AAF) [20] represents the proportion of death that could be prevented if these exposures could be prevented. To assess the 6-month attributable mortality risk associated with each domain while keeping the final model as simple as possible in the spirit of parsimony, we constructed the final model with 2 simple steps: (1) separately selected the most important variables in each of the 4 domains, and (2) fit the final model with all the variables selected in the first step.

Separately for each domain, all variables were included in a multivariate logistic model with 6-month mortality as the explained variable. Odds ratios (ORs), AAFs [20,21], and their corresponding $95 \%$ confidence intervals ( $95 \%$ CIs) were estimated for each variable. For AAFs, CIs were derived with Monte Carlo simulations [20]. The most important variables of each domain-specific multivariate 
logistic model were selected based on their $p$ value $(p<0.05)$. Then, the final multivariate model was constructed with the most important variables of each domain selected in the previous step. No further selection of variables was performed. Again, ORs and AAFs were provided with their $95 \%$ CI.

Because the final model depended on how the variables of each domain were selected, 2 sensitivity analyses were performed: (1) selection of the variables with $p<0.10$ in each domain-specific multivariate logistic model; (2) selection of the 3 variables with the greater AAF in each domain-specific multivariate logistic model.

All models were internally validated with the same approach by using boostrap. Optimism-corrected c-index (i.e., discrimination) and optimism-corrected calibration plots were calculated for each model (FigureS1).

Additional post-hoc statistical analyses were performed to test time trends for mortality, co-existing acute illness, type of fracture, type of surgery, and postoperative complications by Fisher's test for trend in proportions.

All $p$ values are two-tailed, and $p<0.05$ was considered to denote significant difference. Statistical analyses involved using R v3.6.1.

\section{Results}

Our cohort included 1010 patients (Figure 2); baseline characteristics are shown in Table 1. In total, $32(3.2 \%)$ patients died during acute care, $36(3.6 \%)$ during rehabilitation, and $81(8.0 \%)$ after returning to home and/or an institution. The 6-month mortality rate was $14.8 \%$ (95\% CI: 12.6 to 17.1$)$.

We found a significant correlation between the ADL and Rockwood scores $(\mathrm{R}=-0.68, p<0.001)$, and IADL and Rockwood scores $(\mathrm{R}=-0.68, p<0.001)$. The ADL score was retained as the variable assessing autonomy. We found a correlation between the CIRS score and number of medications $(\mathrm{R}=0.57, p<0.001)$, and the CIRS was retained as the variable assessing comorbidities. The optimal threshold of the ADL score to predict 6-month mortality was a score $\geq 5.5$ (sensitivity 0.57 , specificity 0.67 ). Conversely, the best threshold of the CIRS to predict 6-month mortality was a score $\geq 11$ (sensitivity 0.60 , specificity 0.67 ) and that for age was $\geq 88$ years (sensitivity 0.58 , specificity 0.57 ). In a multivariate model including baselines variables, we determined that the age, gender, CIRS score, ADL score, and type of fracture were significantly associated with 6-month mortality after HF (Table S1).

Acute co-existing illness (Table 2) was diagnosed in 105 patients (10.4\%, 95\% CI: 8.6 to 12.4 ). The occurrence of an acute co-existing illness was not significantly associated with 6-month mortality after HF (Table S2).

Most patients underwent surgery before $48 \mathrm{~h}$ after hospital admission (81.0\%, 95\% CI: 79.3 to 84.2 ; median time to surgery: $24(17-44)$ h). Perioperative factors are shown in Table 2. On multivariate logistic regression including all perioperative factors, blood transfusion and time to surgery $>48 \mathrm{~h}$ were associated with 6-month mortality after HF (Table S3 and Figure S2)

Postoperative complications are shown in Table 3; 104 patients $(10.3 \%, 95 \%$ CI 8.5 to 12.4$)$ had at least one severe postoperative complication (Dindo-Clavien score $\geq 3$ ) (Table S4). On multivariate logistic regression, the occurrence of severe postoperative complications was significantly associated with 6-month mortality after HF (OR 5.88, 95\% CI 3.78 to 9.12, $p<0.001$ ) (Figure S2 and Table S5).

Table 4 and Figure 3 show the AAF of the different domains. The weight of baseline characteristics was greatest $(62.4 \%, 95 \%$ CI: 50.0 to $74.7 \%)$. Perioperative factors, especially blood transfusion, and severe postoperative complications accounted for $24.2 \%$ of the 6-month mortality (95\% CI: 9.2 to 39.3). Finally, $13.4 \%$ (95\% CI: 0 to 26.9 ) of the 6-month mortality rate was not explained by these variables. Sensitivity analysis gave similar conclusions (Tables S6 and S7, Figure 3). 
Patients $\geq 70$ years old with hip fracture admitted to the hospital from June 2009

to September 2018

$n=1584$ events

$n=1477$ patients

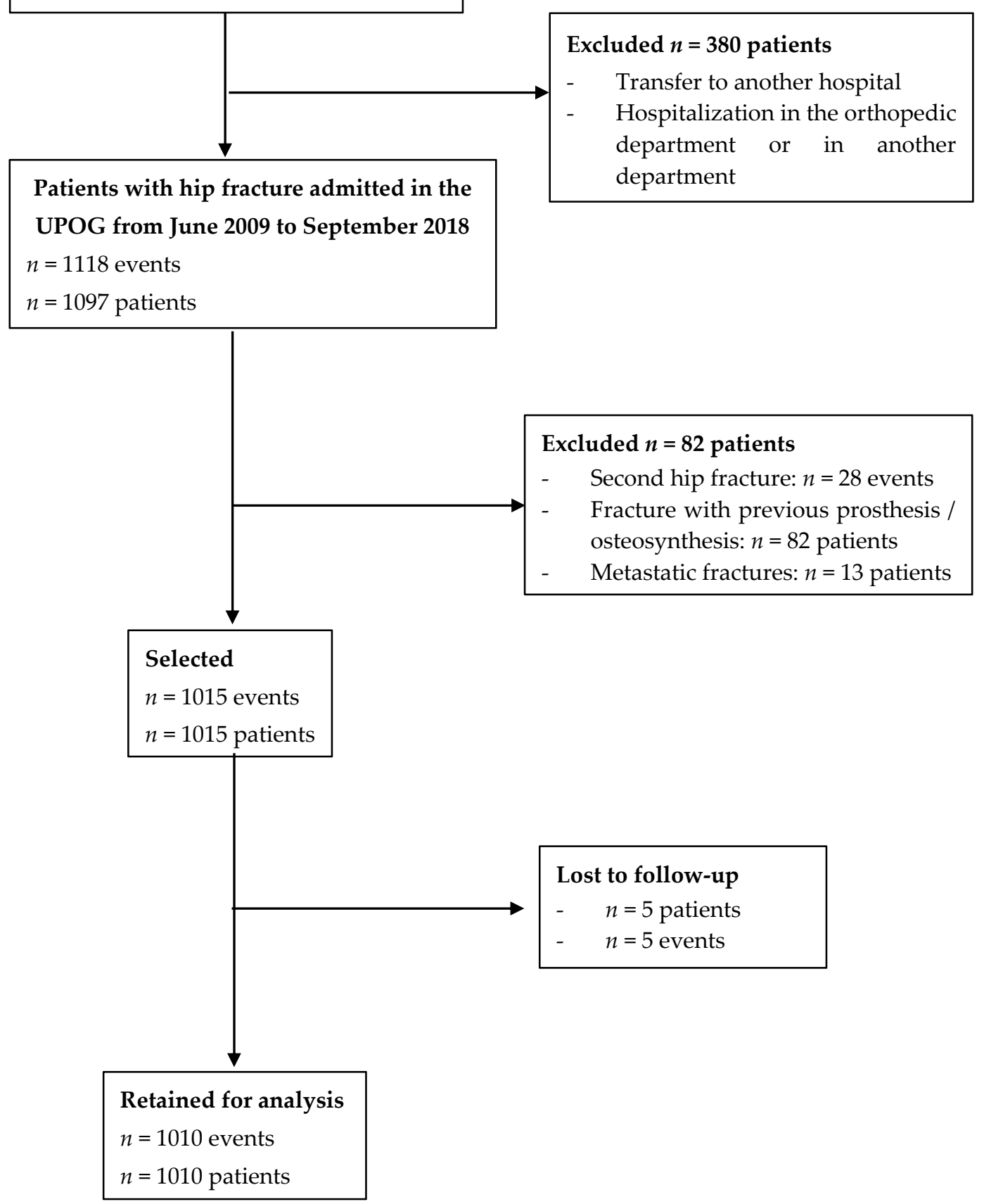

Figure 2. Flow chart. 
Table 1. Baseline characteristics of patients overall and stratified by living status at 6 months after surgery.

\begin{tabular}{|c|c|c|c|c|}
\hline & $\begin{array}{l}\text { All Patients } \\
(n=1010)\end{array}$ & $\begin{array}{l}\text { Deceased } \\
(n=149)\end{array}$ & $\begin{array}{c}\text { Alive } \\
(n=861)\end{array}$ & $p$ Value \\
\hline Age (years) & $86 \pm 6$ & $88 \pm 6$ & $86 \pm 6$ & $<0.001$ \\
\hline Female & $768(76)$ & $99(66)$ & $669(78)$ & 0.003 \\
\hline \multicolumn{5}{|c|}{ Medical history } \\
\hline CIRS score & $9(6-12)$ & $12(9-14)$ & $9(6-12)$ & $<0.001$ \\
\hline CIRS score $\geq 11$ & $378(37)$ & $90(60)$ & $288(33)$ & $<0.001$ \\
\hline Dementia & $394(39)$ & $70(47)$ & $324(38)$ & 0.03 \\
\hline Stroke & $164(16)$ & $32(21)$ & 132 (15) & 0.06 \\
\hline Hypertension & $692(69)$ & $108(72)$ & $584(68)$ & 0.26 \\
\hline Diabetes & $139(14)$ & $26(17)$ & $114(13)$ & 0.24 \\
\hline Atrial fibrillation & $271(27)$ & $72(48)$ & $199(23)$ & 0.44 \\
\hline Coronary artery disease & $183(18)$ & $39(26)$ & $144(17)$ & 0.006 \\
\hline Heart failure & $165(16)$ & $50(34)$ & $115(13)$ & $<0.001$ \\
\hline Obesity $\left(\mathrm{BMI} \geq 30 \mathrm{~kg} / \mathrm{m}^{2)}\right)$ & $66(7)$ & $13(9)$ & $53(6)$ & 0.24 \\
\hline Chronic renal failure ${ }^{a}$ & $115(11)$ & $34(23)$ & $81(9)$ & \\
\hline Missing values & 3 & 0 & 3 & $<0.001$ \\
\hline Cancer & $222(22)$ & $49(33)$ & $173(20)$ & $<0.001$ \\
\hline Number of drugs & $5(3-8)$ & $6(4-9)$ & $5(3-7)$ & $<0.001$ \\
\hline Anticoagulant & $189(19)$ & $44(30)$ & 145 (17) & 0.002 \\
\hline Antiplatelet & $365(36)$ & $53(36)$ & $312(36)$ & 0.87 \\
\hline \multicolumn{5}{|c|}{ Autonomy and frailty } \\
\hline ADL score & $5.5(3.5-6)$ & $4.5(2.5-5.5)$ & $5.5(4-6)$ & $<0.001$ \\
\hline ADL score $>5.5$ & $543(54)$ & $49(33)$ & $494(57)$ & $<0.001$ \\
\hline Rockwood score & $5(4-6)$ & $6(5-6)$ & $5(4-6)$ & $<0.001$ \\
\hline Living in nursing home & $139(14)$ & $27(18)$ & $112(13)$ & 0.09 \\
\hline \multicolumn{5}{|c|}{ Walking ability } \\
\hline Walking without assistance & $410(48)$ & $35(23)$ & $445(44)$ & $<0.001$ \\
\hline Walking with assistance & $544(54)$ & $110(74)$ & $434(50)$ & $<0.001$ \\
\hline Not walking & $17(2)$ & $4(2)$ & $21(2)$ & 0.54 \\
\hline \multicolumn{5}{|c|}{ Fracture } \\
\hline Intertrochanteric fracture & $530(52)$ & $92(62)$ & $438(51)$ & 0.01 \\
\hline Femoral neck fracture & $480(48)$ & $57(38)$ & $423(49)$ & 0.01 \\
\hline
\end{tabular}

Table 2. Co-existing acute illness before surgery and perioperative factors: overall and stratified by living status at 6 months after surgery.

\begin{tabular}{ccccc}
\hline & $\begin{array}{c}\text { All Patients } \\
(\boldsymbol{n}=\mathbf{1 0 1 0})\end{array}$ & $\begin{array}{c}\text { Deceased } \\
(\boldsymbol{n}=\mathbf{1 4 9 )})\end{array}$ & $\begin{array}{c}\text { Alive } \\
(\boldsymbol{n}=\mathbf{8 6 1 )})\end{array}$ & $\boldsymbol{p}$ Value \\
\hline & Acute Co-Existing Illness & & \\
\hline Another associated trauma & $49(5)$ & $2(1)$ & $47(6)$ & 0.003 \\
Any acute co-existing illness & $105(10)^{\mathrm{b}}$ & $16(11)$ & $89(10)$ & 0.88 \\
\hline
\end{tabular}


Table 2. Cont.

\begin{tabular}{|c|c|c|c|c|}
\hline & $\begin{array}{l}\text { All Patients } \\
(n=1010)\end{array}$ & $\begin{array}{c}\text { Deceased } \\
(n=149)\end{array}$ & $\begin{array}{c}\text { Alive } \\
(n=861)\end{array}$ & $p$ Value \\
\hline \multicolumn{5}{|c|}{ Perioperative Factors } \\
\hline \multicolumn{5}{|l|}{ Anesthesia } \\
\hline General anesthesia & $872(96)$ & $130(96)$ & $742(96)$ & 0.80 \\
\hline Missing values & 106 & 14 & 92 & \\
\hline \multicolumn{5}{|l|}{ Surgery } \\
\hline Time to surgery $>48 \mathrm{~h}$ & $192(19)$ & $39(26)$ & $153(18)$ & 0.02 \\
\hline Gamma nail & $513(51)$ & $86(58)$ & $427(50)$ & 0.07 \\
\hline Dynamic hip screw & $65(6)$ & $8(5)$ & $57(7)$ & 0.57 \\
\hline Unipolar prosthesis & $406(40)$ & $54(36)$ & $352(41)$ & 0.28 \\
\hline Bipolar prosthesis & $26(3)$ & $1(0.7)$ & $25(3)$ & 0.16 \\
\hline \multicolumn{5}{|l|}{ Hemoglobin level } \\
\hline Preoperative hemoglobin $\left(\mathrm{g} \cdot \mathrm{dL}^{-1}\right)$ & $12.2 \pm 1.6$ & $11.5 \pm 1.7$ & $12.3 \pm 1.5$ & $<0.001$ \\
\hline In-hospital ${ }^{\mathrm{c}}$ transfusion & $507(50)$ & $100(67)$ & $407(47)$ & $<0.001$ \\
\hline In-hospital ${ }^{\mathrm{C}}$ total packed RBC (unit) & $1(0-2)$ & $2(0-3)$ & $0(0-2)$ & $<0.001$ \\
\hline \multicolumn{5}{|c|}{$\begin{array}{l}\text { Data are mean } \pm \text { SD, median ( } 25-75 \text { interquartile range), or number (percentage). Missing values are detailed only when } \\
\text { they exist. Comparison between the two groups by Mann-Whitney U test for quantitative variables and chi-square test } \\
\text { or Fisher's exact test for qualitative variables. Abbreviations: RBC: red blood cell. a: see text for definition. }{ }^{b} \text { : trauma } \\
\text { lesions }(n=49) \text {, infections }(n=17 \text {, mostly pulmonary, } n=9 \text {, and urinary, } n=5 \text {, infections), acute cardiac disease }(n=12) \text {, } \\
\text { stroke and seizures }(n=7) \text {, blood transfusion }(n=7) \text {, rhabdomyolysis }(n=6) \text { and thromboembolic disease }(n=3) \text {. } \\
\text { The sum of conditions may not add to the total because a patient may have several conditions. }{ }^{c}: \text { i.e., in the emergency } \\
\text { room, surgery, intensive care unit, and perioperative geriatric unit (excluding rehabilitation). }\end{array}$} \\
\hline
\end{tabular}

Table 3. Postoperative factors: overall and stratified by living status at 6 months after surgery.

\begin{tabular}{|c|c|c|c|c|}
\hline & $\begin{array}{l}\text { All Patients } \\
(n=1010)\end{array}$ & $\begin{array}{c}\text { Deceased } \\
(n=149)\end{array}$ & $\begin{array}{c}\text { Alive } \\
(n=861)\end{array}$ & $p$ Value \\
\hline \multicolumn{5}{|c|}{ In-hospital ${ }^{a}$ postoperative complications } \\
\hline Dindo-Clavien score & $2(1-2)$ & $2(2-4)$ & $2(1-2)$ & $<0.001$ \\
\hline Dindo-Clavien score $\geq 3$ & $104(10)$ & $45(30)$ & $59(7)$ & $<0.0001$ \\
\hline Postoperative delirium & $404(40)$ & $79(53)$ & $325(38)$ & 0.004 \\
\hline Atrial fibrillation & $83(8)$ & $19(13)$ & $64(7)$ & 0.03 \\
\hline Acute coronary syndrome & $83(8)$ & $25(17)$ & $58(7)$ & $<0.001$ \\
\hline Acute heart failure & $117(12)$ & $43(29)$ & $74(9)$ & $<0.001$ \\
\hline Venous thromboembolic event & $44(4)$ & $6(4)$ & $38(4)$ & 0.83 \\
\hline Hemorrhage & $93(9)$ & $21(14)$ & $72(8)$ & 0.03 \\
\hline Infection & $168(17)$ & $44(30)$ & $124(14)$ & $<0.001$ \\
\hline Surgical revision & $19(2)$ & $4(3)$ & $15(2)$ & 0.51 \\
\hline Bladder retention & $268(27)$ & $50(34)$ & $218(25)$ & 0.04 \\
\hline Stool impaction & $448(44)$ & $76(51)$ & $372(43)$ & 0.07 \\
\hline Pressure sore & $110(11)$ & $34(23)$ & $76(9)$ & $<0.001$ \\
\hline Admission to ICU & $47(5)$ & $20(13)$ & $27(3)$ & $<0.001$ \\
\hline \multicolumn{5}{|c|}{ Walking ability } \\
\hline Time to first sitting (days) & $1(1-2)$ & $2(1-3)$ & $1(1-2)$ & \\
\hline Missing values & 20 & 7 & 13 & 0.001 \\
\hline Time to first walking (days) & $2(1-3)$ & $2(1-4)$ & $2(1-3)$ & \\
\hline Missing values & 77 & 22 & 55 & 0.007 \\
\hline \multicolumn{5}{|c|}{ At discharge } \\
\hline Home $^{\mathrm{b}}$ & $152(15)$ & $19(13)$ & $133(15)$ & 0.39 \\
\hline Rehabilitation care & $814(81)$ & $93(63)$ & $721(84)$ & \\
\hline Missing values & 1 & 1 & 0 & $<0.001$ \\
\hline
\end{tabular}

Data are mean \pm SD, median (25-75 interquartile range), or number (percentage). Missing values are detailed only when they exist. Comparison between the two groups by Mann-Whitney U test for quantitative variables and chi-square test or Fisher's exact test for qualitative variables. Abbreviations: ICU: intensive care unit. ${ }^{a}$ : i.e., in the emergency room, surgery, ICU, and perioperative geriatric unit (excluding rehabilitation). ${ }^{\mathrm{b}}$ : home includes institution if the patient was previously in an institution. 
Table 4. Estimates of the 6-month attributable mortality for each domain.

\begin{tabular}{|c|c|c|c|}
\hline Variables & OR $(95 \% \mathrm{CI})$ & $p$ Value & AAF (\%) $(95 \%$ CI) \\
\hline \multicolumn{4}{|c|}{ Baseline characteristics $\rightarrow$ AAF $=62.4$} \\
\hline $\begin{array}{l}\text { Age, ref }<88 \text { years } \\
-\quad \text { Age } \geq 88 \text { years }\end{array}$ & $1.72(1.16-2.56)$ & 0.007 & $10.8(2.9-18.7)$ \\
\hline $\begin{array}{l}\text { Gender, ref }=\text { Female } \\
-\quad \text { Gender }=\text { Male }\end{array}$ & $1.93(1.25-2.97)$ & 0.003 & $7.3(1.4-13.1)$ \\
\hline $\begin{array}{l}\text { CIRS score, ref }<11 \\
\text { - } \quad \text { CIRS score } \geq 11\end{array}$ & $2.27(1.55-3.35)$ & $<0.001$ & $15.6(6.1-25.0)$ \\
\hline $\begin{array}{l}\text { ADL score, ref } \geq 5.5 \\
\bullet \quad \text { ADL score }<5.5\end{array}$ & $2.52(1.68-3.76)$ & $<0.001$ & $20.0(9.8-30.2)$ \\
\hline $\begin{array}{l}\text { Femoral neck fracture, } \text { ref }=\text { No } \\
\text { - } \quad \text { Femoral neck fracture }=\text { Yes }\end{array}$ & $0.67(0.45-1.01)$ & 0.054 & $8.7(0.1-17.2)$ \\
\hline \multicolumn{4}{|c|}{$\begin{array}{l}\text { Co-existing acute illness } \rightarrow \mathrm{AAF}=0 \\
\text { Perioperative factors } \rightarrow \mathrm{AAF}=12.3\end{array}$} \\
\hline \multirow{2}{*}{$\begin{array}{l}\text { Time to surgery, ref } \leq 48 \mathrm{~h} \\
\text { - Time to surgery }>48 \mathrm{~h} \\
\text { Transfusion, ref = No } \\
\text { - Transfusion = yes }\end{array}$} & $1.36(0.87-2.13)$ & 0.18 & $2.7(1.8-7.3)$ \\
\hline & $1.53(1.02-2.28)$ & 0.04 & $9.6(1.3-20.5)$ \\
\hline \multicolumn{4}{|c|}{ Postoperative factors $\rightarrow$ AAF $=11.9$} \\
\hline \multirow[t]{2}{*}{$\begin{array}{l}\text { Dindo-Clavien score, ref }<3 \\
\text { - } \quad \text { Dindo-Clavien score } \geq 3\end{array}$} & $4.91(3.06-7.90)$ & $<0.001$ & $11.9(6.9-16.9)$ \\
\hline & & Total & $86.6(73.1-100)$ \\
\hline
\end{tabular}

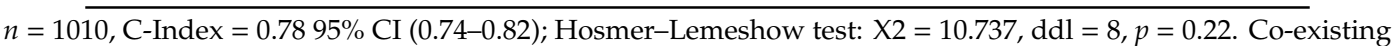
acute illness factors were not included in the final model because they were not significant on univariate analysis. Abbreviations: $\mathrm{AAF}=$ averaged attributable fraction, ADL: Activities of Daily Living, CIRS: Cumulative Illness Rating Scale; OR = odds ratio, CI: confidence interval; ref = reference value.

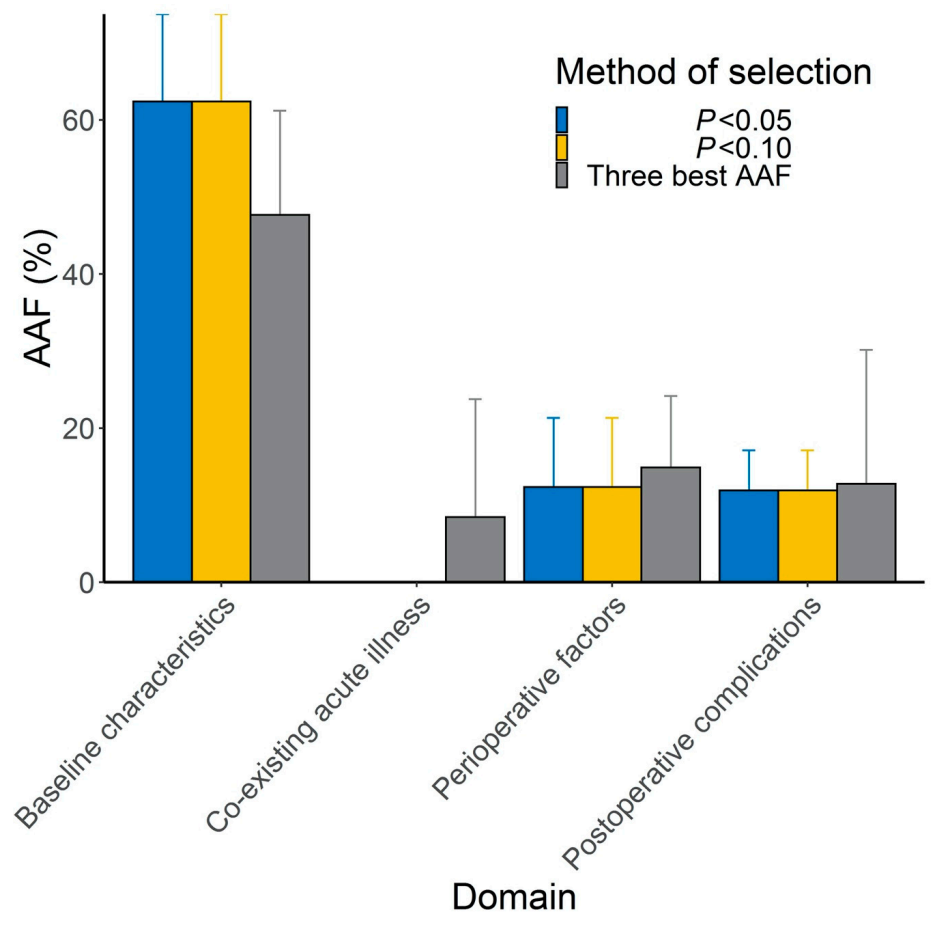

Figure 3. Results of 3 analyses of the estimation of attributable mortality (scale from 0 to 80 ) associated with baseline characteristics, co-existing acute illness, perioperative factors, and severe postoperative complications. The main analysis was selection of the variables with $\mathrm{p}<0.05$ for each domain-specific multivariate logistic model and the 2 others were considered sensitivity analyses. The whiskers represent the upper limit of the bilateral 95\% confidence interval. Abbreviation: AAF = averaged attributable fraction. 
Additional post-hoc statistical analyses revealed no significant difference over time in 6-month mortality rate $(p=0.80)$, rate of co-existing acute illnesses $(p=0.40)$, type of fracture $(p=0.40)$, and rate of postoperative complications $(p=0.70)$.

\section{Discussion}

In estimating the respective influence of a priori selected risk factors on six-month mortality after $\mathrm{HF}$, baseline characteristics were the most important contributing factors (62.4\%, 95\% CI: 50.0 to $74.7 \%$ ). Postoperative complications (11.9\%, 95\% CI: 6.9 to $16.9 \%)$, perioperative blood transfusion $(9.6 \%, 95 \%$ CI: 1.3 to $20.5 \%)$ and delayed surgery (2.7\%, $95 \%$ CI: 1.8 to $7.3 \%)$ had lower but still significant weight. Our results, estimating for the first time the respective influence of a priori selected risk factors on six-month mortality after HF, suggest that a maximum of $24.2 \%$ of deaths could be avoided if all of these modifiable factors could be prevented.

Baseline characteristics explained less than two-thirds of the six-month mortality. This result helps in understanding why preoperative scores are inaccurate to predict mortality [8] and why mortality after HF is six times that observed after elective total hip replacement, even when patients are matched for baseline characteristics [10]. Although this study was retrospective, its observational nature allowed us to include more patients with preoperative severe baseline characteristics than what we could observe in a randomized controlled trial. Indeed, clinical trials feature an under-representation of older adults, particularly those with multimorbidity and polypharmacy, as compared with actual conditions of medicine use in real-world practice [22]. We were able to obtain a lot of information concerning baseline characteristics of our patients, in line with a geriatric point of view that tries to assess all variables that characterize the geriatric patient versus healthy aging [23]. However, we observed high correlations between these variables, and we finally retained only age and the CIRS score for comorbidities and the ADL score for autonomy.

Delayed surgery was not an important issue (estimates of the six-month attributable mortality: $2.7 \%$ ), which contrasts with previous studies. A meta-analysis including 191,873 patients [12] found that early surgery (cut-off between 24 and $48 \mathrm{~h}$ ) was associated with significantly reduced risk of death (OR $0.74 ; 95 \%$ CI: $0.67-0.81$ ). Nevertheless, this previous observational study failed to identify patients with surgery delayed for valid medical reasons, who are thought to have a poorer outcome, thereby suggesting possible confounders. In our study, only $19 \%$ of the patients had delayed surgery per this definition, so delay may not be important when HF is considered an urgent surgical procedure and when only a limited number of patients undergo surgery later. However, in most countries, including France, approximately half of the older patients with HF undergo surgery with a delay $>48 \mathrm{~h}$ (mainly because of preoperative medical assessment and access to the operating room) [8,24]. A recent large randomized trial failed to observe a significant decrease in mortality when comparing early (median $6 \mathrm{~h}$ ) vs. delayed (median $24 \mathrm{~h}$ ) surgery. Our results are consistent with the non-significant estimate of risk reduction $(1 \%, p=0.40)$ [25].

The type of anesthesia used was not associated with outcome in our study, which agrees with findings from a recent large observational study [26]. Nevertheless, because most of our patients had general anesthesia (96\%), our study lacks the power to answer this question. We assessed the perioperative period with only blood transfusion and hemoglobin values, thereby failing to precisely assess perioperative hemodynamic stability. However, there are few data to indicate that intraoperative instability may have a major impact on outcome as compared to other known factors in this population. In a recent meta-analysis of five studies including a total of 403 participants, perioperative hemodynamic optimization did not significantly improve outcomes for older patients with HF [27]. In addition, in a recent multicenter clinical trial of patients predominantly undergoing major abdominal surgery, management targeting individualized blood pressure as compared with standard management reduced the risk of postoperative organ dysfunction but not 30-day mortality [28].

Postoperative complications represent some important factors associated with mortality. This result is consistent with a previous study showing that $57 \%$ of in-hospital deaths in older patients with 
HF may be preventable [29]. This result is also impressive when considering that our study was performed in a dedicated environment where mortality is reduced. ${ }^{3}$ Thus, future research should focus on preventing these complications. Our study suggests that swallowing disorders, postoperative delirium, pressure sores, heart failure, acute myocardial infarction, and atrial fibrillation, among other factors, might require particular attention.

\section{Limitations}

This was an observational study, and causality cannot be demonstrated. Although we considered as much information as possible and used advanced statistical methods to correct for confounding biases, we can only assume that all relevant confounders have been identified. Moreover, the variable selection approach used could be a limitation to the generalization of our findings. However, our sensitivity analyses gave similar results.

More importantly, $13.4 \%$ of the deaths observed at six months were not explained by this model, meaning by one of the four domains. Similar results are common in most other clinical predictive models. This finding suggests that our models' predictive performances were excellent but not perfect. The most relevant interpretation is that some predictors were omitted in the models (i.e., not recorded or unknown predictors), that all events (death) were not preventable, or also that our parsimonious modeling approach wasted a part of the information. An example of omitted predictors could be a defect in immune regulation, as it has been reported in a model of septic acute stress [30]. Indeed, an increase in inflammatory markers has been reported after HF and was associated with post-operative mortality [31,32]. However, while not perfect, the predictive performance of our model supported of modelling approach and allowed us to provide meaningful clinical interpretations without a significant level of imprecision.

Our study was conducted in a highly specialized environment that is associated with reduced six-month mortality as compared with patients admitted to orthopedic departments [9]. Therefore, our results may not be extrapolated to conventional or other orthogeriatric models previously reported.

\section{Conclusions}

Baseline characteristics of the older patients explained less than two-thirds of the six-month mortality after HF surgery. Optimizing the care of older patients with HF, by improving management of perioperative factors (postoperative complications, perioperative blood transfusion, and delayed surgery) could reduce by a maximum of one quarter the six-month mortality rate after HF. These results, providing new information to help design future research at the forefront of care improvement for older patients with hip fracture, indicate that physicians should focus on early detection and treatment of severe postoperative complications. In addition, a $13.4 \%$ mortality rate was not explained by our model, indicating that there are still unknown predictive factors.

Supplementary Materials: The following are available online at http://www.mdpi.com/2077-0383/9/8/2370/s1. Text S1: STROBE Statement-Checklist of items that should be included in reports of cohort studies. Text S2: Management in UPOG. Text S3: Original statistical analysis plan. Figure S1: Apparent and optimism-corrected calibration plots for the internal validation of the 3 models. Figure S2: Non-adjusted survival curves for death in patients. Table S1: The intermediate multivariate logistic model predicting 6-month mortality with baseline characteristics. Table S2: The intermediate multivariate logistic model predicting 6-month mortality with acute co-existing illness. Table S3: The intermediate multivariate logistic model predicting 6-month mortality with perioperative factors. Table S4: Complications quoted as severe (Dindo-Clavien score 3 to 5) and observed during the acute stay $(n=104)$. Table S5: The intermediate multivariate logistic model predicting 6-month mortality with postoperative complications. Table S6: Estimates of the 6-month attributable mortality according to each domain (sensitivity analyses: selection of the variables with $p<0.10$ for each domain-specific multivariate logistic model). Table S7: Estimates of the 6-month attributable mortality according to each domain (sensitivity analyses: selection of the 3 variables with the greater AAF in each domain-specific multivariate logistic model).

Author Contributions: Conceptualization, L.Z., D.H., Y.L.M., B.R., and J.B.; formal analysis, D.H.; methodology, L.Z., D.H., Y.L.M., B.R., and J.B.; resources, L.Z., M.R., J.C.-B., A.M., F.K., and J.B.; software, L.Z.; supervision, B.R. and J.B.; validation, B.R. and J.B.; writing-original draft, L.Z.; critical revision of the manuscript for important 
intellectual content: all authors. J.B. had full access to all data in the study and takes responsibility for the integrity and the accuracy of the data; D.H., public health physician and statistician, performed the statistical analyses and was an independent statistician who was not involved in data collection. All authors have read and agreed to the published version of the manuscript.

Funding: This work was supported only by institutional sources

Acknowledgments: We thank David Baker, DM, FRCA, (Department of Anesthesiology and Critical Care, Hôpital Necker-Enfants Malades, Paris) and Laura Smales (BioMedEditing, Toronto, Canada) for reviewing the manuscript.

Conflicts of Interest: All authors have completed and submitted the ICMJE Form for Disclosure of Potential Conflict of Interest. Boddaert reports receiving fees for lectures from VIFOR Pharma and Baxter companies (outside the submitted work). No other disclosures were reported.

\section{References}

1. Berry, S.D.; Kiel, D.P.; Colón-Emeric, C. Hip fractures in older adults in 2019. JAMA 2019, 321, $2231-2232$. [CrossRef] [PubMed]

2. Brauer, C.A.; Coca-Perraillon, M.; Cutler, D.M.; Rosen, A.B. Incidence and mortality of hip fractures in the United States. JAMA 2009, 302, 1573-1579. [CrossRef] [PubMed]

3. Johnell, O.; Kanis, J.A. An estimate of the worldwide prevalence and disability associated with osteoporotic fractures. Osteoporos. Int. 2006, 17, 1726-1733. [CrossRef] [PubMed]

4. Goldacre, M.J.; Roberts, S.E.; Yeates, D. Mortality after admission to hospital with fractured neck of femur: Database study. BMJ 2002, 325, 868-869. [CrossRef]

5. Hannan, E.L.; Magaziner, J.; Wang, J.J.; Eastwood, E.A.; Silberzweig, S.B.; Gilbert, M.; Morrison, R.S.; McLaughlin, M.A.; Orosz, G.M.; Siu, A.L. Mortality and locomotion 6 months after hospitalization for hip fracture: Risk factors and risk-adjusted hospital outcomes. JAMA 2001, 285, 2736-2742. [CrossRef]

6. Cram, P.; Lu, X.; Kaboli, P.J.; Vaughan-Sarrazin, M.S.; Cai, X.; Wolf, B.R.; Li, Y. Clinical characteristics and outcomes of Medicare patients undergoing total hip arthroplasty, 1991-2008. JAMA 2011, 305, 1560-1567. [CrossRef]

7. Roche, J.J.W.; Wenn, R.T.; Sahota, O.; Moran, C.G. Effect of comorbidities and postoperative complications on mortality after hip fracture in elderly people: Prospective observational cohort study. BMJ 2005, 331, 1374. [CrossRef]

8. Boddaert, J.; Raux, M.; Khiami, F.; Riou, B. Perioperative management of elderly patients with hip fracture. Anesthesiology 2014, 121, 1336-1341. [CrossRef]

9. Boddaert, J.; Cohen-Bittan, J.; Khiami, F.; Le Manach, Y.; Raux, M.; Beinis, J.-Y.; Verny, M.; Riou, B. Postoperative admission to a dedicated geriatric unit decreases mortality in elderly patients with hip fracture. PLOS ONE 2014, 9, e83795. [CrossRef]

10. Le Manach, Y.; Collins, G.; Bhandari, M.; Bessissow, A.; Boddaert, J.; Khiami, F.; Chaudhry, H.; De Beer, J.; Riou, B.; Landais, P.; et al. Outcomes after hip fracture surgery compared with elective total hip replacement. JAMA 2015, 314, 1159-1166. [CrossRef]

11. Boddaert, J.; Na, N.; Le Manach, Y.; Raux, M.; Cohen-Bittan, J.; Vallet, H.; Meziere, A.; Khiami, F.; Riou, B. Prediction of postoperative mortality in elderly patients with hip fracture: Are specific and geriatric scores better than general scores? Br. J. Anaesth 2017, 118, 952-954. [CrossRef]

12. Moja, L.; Piatti, A.; Pecoraro, V.; Ricci, C.; Virgili, G.; Salanti, G.; Germagnoli, L.; Liberati, A.; Banfi, G. Timing matters in hip fracture surgery: Patients operated within 48 hours have better outcomes. A meta-analysis and meta-regression of over 190,000 patients. PLoS ONE 2012, 7, e46175. [CrossRef] [PubMed]

13. Von Elm, E.; Altman, D.G.; Egger, M.; Pocock, S.J.; Gøtzsche, P.C.; Vandenbroucke, J.P. STROBE Initiative the Strengthening the Reporting of Observational Studies in Epidemiology (STROBE) statement: Guidelines for reporting observational studies. PLoS Med. 2007, 4, e296. [CrossRef] [PubMed]

14. Collins, G.S.; Reitsma, J.B.; Altman, D.G.; Moons, K.G.M. Transparent Reporting of a multivariable prediction model for Individual Prognosis or Diagnosis (TRIPOD): The TRIPOD statement. Ann. Intern. Med. 2015, 162, 55-63. [CrossRef] [PubMed]

15. Linn, B.S.; Linn, M.W.; Gurel, L. Cumulative illness rating scale. J. Am. Geriatr. Soc. 1968, 16, 622-626. [CrossRef] [PubMed]

16. Rockwood, K.; Song, X.; MacKnight, C.; Bergman, H.; Hogan, D.B.; McDowell, I.; Mitnitski, A. A global clinical measure of fitness and frailty in elderly people. CMAJ 2005, 173, 489-495. [CrossRef] 
17. Katz, S.; Akpom, C.A. 12. Index of ADL. Med. Care 1976, 14, 116-118. [CrossRef]

18. Lawton, M.P.; Brody, E.M. Assessment of older people: Self-maintaining and instrumental activities of daily living. Gerontologist 1969, 9, 179-186. [CrossRef]

19. Dindo, D.; Demartines, N.; Clavien, P.-A. Classification of surgical complications: A new proposal with evaluation in a cohort of 6336 patients and results of a survey. Ann. Surg. 2004, 240, 205-213. [CrossRef]

20. Ferguson, J.; Alvarez-Iglesias, A.; Newell, J.; Hinde, J.; O’Donnell, M. Estimating average attributable fractions with confidence intervals for cohort and case-control studies. Stat. Methods Med. Res. 2018, 27, 1141-1152. [CrossRef]

21. Eide, G.E.; Gefeller, O. Sequential and average attributable fractions as aids in the selection of preventive strategies. J. Clin. Epidemiol. 1995, 48, 645-655. [CrossRef]

22. Thake, M.; Lowry, A. A systematic review of trends in the selective exclusion of older participant from randomised clinical trials. Arch. Gerontol. Geriatr. 2017, 72, 99-102. [CrossRef] [PubMed]

23. Guiding principles for the care of older adults with multimorbidity: An approach for clinicians Guiding principles for the care of older adults with multimorbidity: An approach for clinicians: American Geriatrics Society Expert Panel on the Care of Older Adults with Multimorbidity. J. Am. Geriatr. Soc. 2012, 60, E1-E25. [CrossRef]

24. White, S.M.; Griffiths, R.; Holloway, J.; Shannon, A. Anaesthesia for proximal femoral fracture in the UK: First report from the NHS Hip Fracture Anaesthesia Network. Anaesthesia 2010, 65, 243-248. [CrossRef]

25. HIP ATTACK Investigators Accelerated surgery versus standard care in hip fracture (HIP ATTACK): An international, randomised, controlled trial. Lancet 2020, 395, 698-708. [CrossRef]

26. Neuman, M.D.; Rosenbaum, P.R.; Ludwig, J.M.; Zubizarreta, J.R.; Silber, J.H. Anesthesia technique, mortality, and length of stay after hip fracture surgery. JAMA 2014, 311, 2508-2517. [CrossRef]

27. Lewis, S.R.; Butler, A.R.; Brammar, A.; Nicholson, A.; Smith, A.F. Perioperative fluid volume optimization following proximal femoral fracture. Cochrane Database Syst Rev. 2016, 3, CD003004. [CrossRef]

28. Futier, E.; Lefrant, J.-Y.; Guinot, P.-G.; Godet, T.; Lorne, E.; Cuvillon, P.; Bertran, S.; Leone, M.; Pastene, B.; Piriou, V.; et al. Effect of Individualized vs standard blood pressure management strategies on postoperative organ dysfunction among high-risk patients undergoing major surgery: A randomized clinical trial. JAMA 2017, 318, 1346-1357. [CrossRef]

29. Tarrant, S.M.; Hardy, B.M.; Byth, P.L.; Brown, T.L.; Attia, J.; Balogh, Z.J. Preventable mortality in geriatric hip fracture inpatients. Bone Joint J. 2014, 96-B, 1178-1184. [CrossRef]

30. Delano, M.J.; Ward, P.A. The immune system's role in sepsis progression, resolution, and long-term outcome. Immunol. Rev. 2016, 274, 330-353. [CrossRef]

31. Vallet, H.; Chenevier-Gobeaux, C.; Villain, C.; Cohen-Bittan, J.; Ray, P.; Epelboin, L.; Verny, M.; Riou, B.; Khiami, F.; Boddaert, J. Prognostic value of serum procalcitonin after orthopedic surgery in the elderly population. J. Gerontol. A Biol. Sci. Med. Sci. 2017, 72, 438-443. [CrossRef] [PubMed]

32. Larsen, M.; Bayard, C.; Lepetitcorps, H.; Cohen-Bittan, J.; Appay, V.; Boddaert, J.; Sauce, D. Elevated neopterin levels predict early death in older hip-fracture patients. EBioMedicine 2017, 26, 157-164. [CrossRef] [PubMed] 